ARTICLE

\title{
Atomically resolved three-dimensional structures of electrolyte aqueous solutions near a solid surface
}

Daniel Martin-Jimenez ${ }^{1}$, Enrique Chacon ${ }^{1}$, Pedro Tarazona ${ }^{2} \&$ Ricardo Garcia ${ }^{1}$

Interfacial liquid layers play a central role in a variety of phenomena ranging from friction to molecular recognition. Liquids near a solid surface form an interfacial layer where the molecular structure is different from that of the bulk. Here we report atomic resolution three-dimensional images of electrolyte solutions near a mica surface that demonstrate the existence of three types of interfacial structures. At low concentrations (0.01-1 M), cations are adsorbed onto the mica. The cation layer is topped by a few hydration layers. At higher concentrations, the interfacial layer extends several nanometres into the liquid. It involves the alternation of cation and anion planes. Fluid Density Functional calculations show that water molecules are a critical factor for stabilizing the structure of the interfacial layer. The interfacial layer stabilizes a crystal-like structure compatible with liquid-like ion and solvent mobilities. At saturation, some ions precipitate and small crystals are formed on the mica.

\footnotetext{
${ }^{1}$ Instituto de Ciencia de Materiales de Madrid, CSIC, c/ Sor Juana Ines de la Cruz 3, 28049 Madrid, Spain. 2 Department Física Teórica de la Materia Condensada, IFIMAC Condensed Matter Physics Center, UAM, 28049 Madrid, Spain. Correspondence and requests for materials should be addressed to R.G. (email: r.garcia@csic.es).
} 
nterfacial liquid layers are involved in a wide range of phenomena such as wetting ${ }^{1,2}$, adhesion ${ }^{3}$, surface electrochemistry ${ }^{4}$, nanorheology $y^{5}$, nanolithography ${ }^{6}$, biomineralization $^{7}$, protein dynamics ${ }^{8}$ or protein folding and molecular recognition ${ }^{9}$. It is known that liquids near a solid surface form an interfacial layer where the molecular structure is different from the organization of molecules in the bulk ${ }^{10,11}$. The structure and the associated dynamics of the layer are controlled by the discrete character of the ions and solvent molecules. The number density of liquid molecules next to a solid surface might oscillate with respect to the bulk density and with a periodicity close to one molecular diameter. Those oscillations have been studied with computer simulations ${ }^{12}$ and Classical Fluid Density Functional Theory $(\mathrm{CF}-\mathrm{DFT})^{13,14}$. The oscillations could extend several molecular diameters into the liquid and give rise to a periodic force when two surfaces confining the liquid are brought together into a few molecular diameter thick gaps ${ }^{15-17}$

More than 30 years have passed since the first observation of periodic hydration forces between mica surfaces in dilute electrolyte solutions ${ }^{15}$. A variety of experimental and theoretical methods have been applied to study interfacial liquids, including $\mathrm{X}$-ray reflectivity ${ }^{18}$, X-ray absorption spectroscopy ${ }^{19}$, various force spectroscopies ${ }^{17,20,21}$, first-principle ${ }^{22}$ and molecular dynamics calculations ${ }^{23,24}$. In particular, electronic DFT simulations have shown that a three-dimensional (3D) atomic structure should appear at the liquid adjacent to a solid crystalline surface ${ }^{25}$; however, to this date its direct experimental observation has remained elusive. Recent developments in highresolution atomic force microscopy (AFM) have imaged the existence of a few hydration layers on top of a mica surface ${ }^{26,27}$, ionic crystals ${ }^{28,29}$, lipid headgroups ${ }^{30}$ and protein surfaces ${ }^{31}$. AFM images have also revealed with atomic resolution the adsorption from a solution of $\mathrm{Na}^{+}, \mathrm{Rb}^{+}, \mathrm{Mg}^{2+}$ or $\mathrm{Ca}^{2+}$ ions on different surfaces ${ }^{32-35}$. Those results indicate that advanced force microscopy methods could be applied to determine the 3D atomic structure of liquids near solid surfaces.

Muscovite mica is a suitable substrate to study the interfacial structure of alkali halide aqueous (aq.) solutions because it is atomically flat and it has a net negative surface charge on cleavage. The electrostatic interaction of the alkali cations with mica would favour their attachment. The basal plane (cleaved) of the mica (001) has a hexagonal structure similar to the (111) planes of $\mathrm{NaCl}$ and $\mathrm{KCl}$ crystals. The mica lattice constant in the (001) plane $(0.52 \mathrm{~nm})$ is higher than the corresponding for $\mathrm{NaCl}(111)$ and $\mathrm{KCl}(111), 0.398$ and $0.444 \mathrm{~nm}$, respectively.

Here we provide atomically resolved $3 \mathrm{D}$ images of solidelectrolyte interfaces with several nanometres in depth. The images acquired at $300 \mathrm{~K}$ allow us to characterize the interfacial structures as a function of the electrolyte concentration near a mica surface. At low-to-moderate molarities $(<1 \mathrm{M})$, cations are adsorbed on mica ${ }^{33}$. The monolayer of cations is topped by a few hydration layers. At high molarities, the interfacial layer shows an epitaxial-like growth structure that extends several nanometres into the liquid. The ordered layer involves an alternation of cation and anion planes. A significant number of water molecules are placed between the planes to balance the electrostatic interactions and stabilize the structure. We show that a stable crystalline-like structure is compatible with liquid-like ion and solvent mobilities. At saturation, some ions precipitate and form nano and micro crystals on the mica.

\section{Results}

3D AFM. Figure 1a shows a photograph of the fluid cell. An electrolyte aqueous solution is confined between a mica surface and a glass surface (top of the panel). The AFM cantilever is a

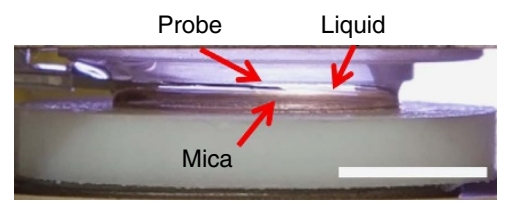

b

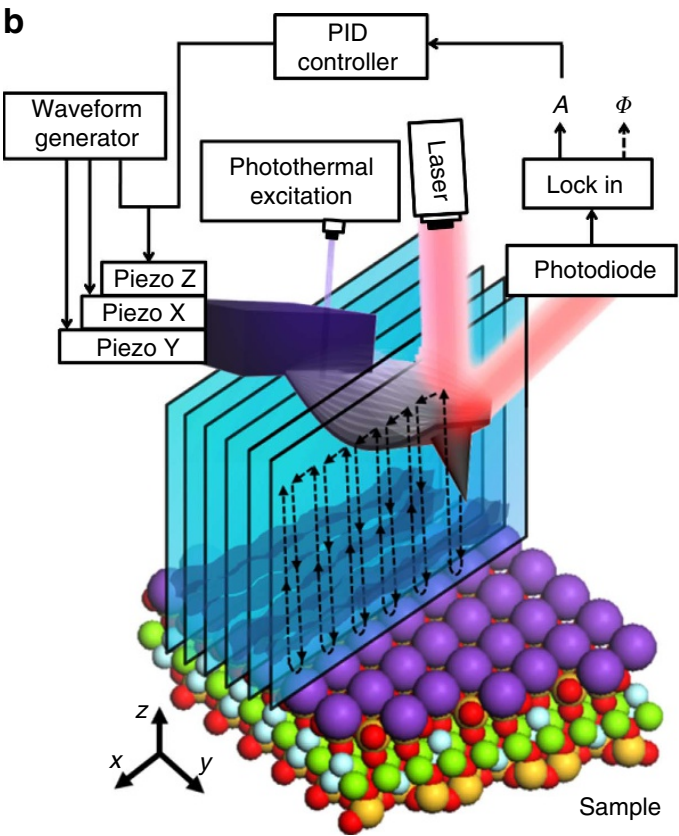

Figure 1 | Fluid cell and 3D AFM scheme. (a) AFM fluid cell. A thin mica substrate is glued onto a Teflon plate (white disk) while a transparent glass surface closes the top of the liquid cell. The thickness of the electrolyte solution is about $1-2 \mathrm{~mm}$. The AFM cantilever is inside the liquid.

(b) Scheme of the tip displacements, cantilever excitation and detection and observables used to acquire a 3D image of a solid-liquid interface. Scale bar, $5 \mathrm{~mm}$ (a).

immersed in the liquid (not seen). The tip explores the 3D solid-liquid interface by acquiring a sequential series of $x z$ planes. In each plane, the tip follows the trajectory depicted in Fig. 1b. The AFM was operated with an amplitude modulation $\mathrm{AFM}^{36}$ feedback control (see Methods section). Amplitude modulation AFM offers a robust and sensitive feedback mechanism against changes in the tip environment. The implementation of 3D AFM with an amplitude modulation feedback has been crucial to expand the depth of the $3 \mathrm{D}$ AFM images to the $10 \mathrm{~nm}$ range. This is in contrast with previous $3 \mathrm{D}$ AFM images ${ }^{26,30}$. Those images were obtained with a frequency modulation feedback where the $3 \mathrm{D}$ range was in the sub- $2 \mathrm{~nm}$ range ${ }^{26,30}$. A $3 \mathrm{D}$ AFM image is obtained by plotting the changes of the tip observables, either the amplitude $(A)$ or the phase shift $(\phi)$, as a function of the spatial coordinates $(x, y, z)$.

Several 3D AFM images of the mica-electrolyte solution interfaces, two corresponding to different mica-KCl (aq.) interfaces and the third to a mica- $\mathrm{NaCl}$ (aq.) interface are shown in Fig. 2. The whole sequence of images is showed in the Supplementary Information (Supplementary Movies 1, 2 and 3). Figure 2a shows an image of a mica-KCl aqueous interface $(0.2 \mathrm{M} \mathrm{KCl})$. The $3 \mathrm{D}$ AFM shows an atomic scale corrugation on the mica (light red region) and an alternation of ultrathin layers $(\sim 0.3 \mathrm{~nm})$ following the atomic corrugation. Calculations (below) assign the atomic corrugation to a monolayer of cations adsorbed on the mica while the ultrathin layers represent variations of the water density. Figure $2 \mathrm{~b}$ shows an image of $\mathrm{a}$ mica- $\mathrm{KCl}$ (aq.) interface $(\sim 4 \mathrm{M} \mathrm{KCl})$. The image shows an 

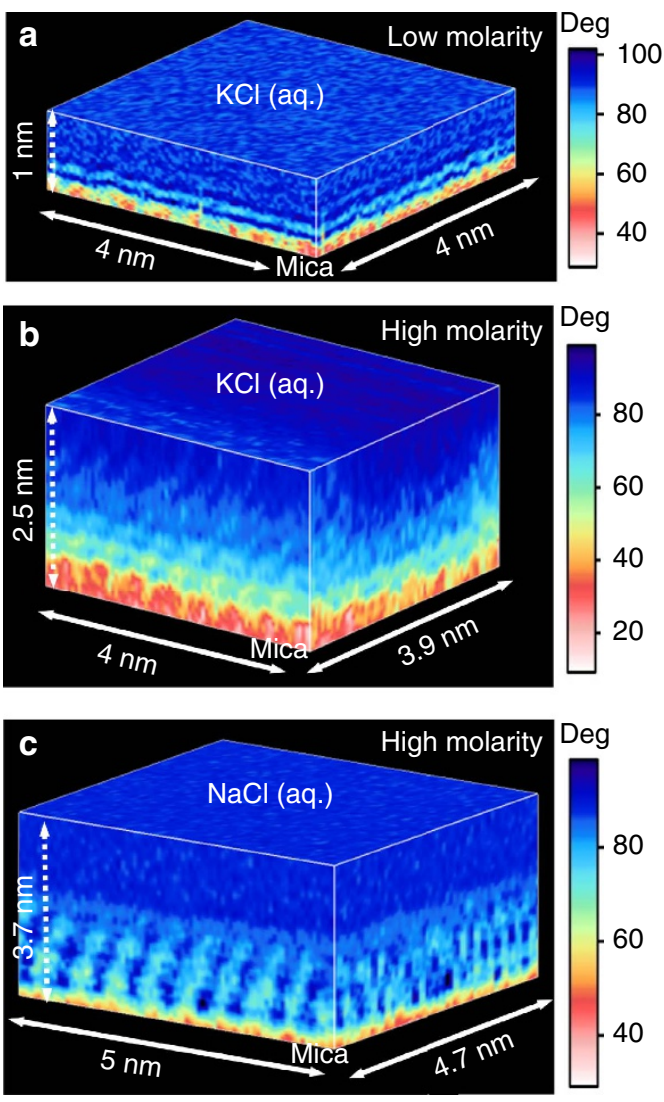

Figure 2 | Three-dimensional images of mica-electrolyte solution interfaces. (a) 3D AFM image of a $\mathrm{KCl}$ (aq.) solution $(0.2 \mathrm{M} \mathrm{KCl})$. The image shows a monolayer of adsorbed $\mathrm{K}^{+}$ions (light red) topped by two hydration layers (lighter stripes). The hydration layers ( $\sim 0.3 \mathrm{~nm}$ thick) follow the atomic corrugation of the surface. (b) 3D AFM image of a mica- $\mathrm{KCl}$ (aq.) interface ( $\sim 4 \mathrm{M})$. The interface is divided in two main regions, an ordered liquid layer extending up to $2 \mathrm{~nm}$ from the mica and the bulk solution above it. (c) 3D AFM image of a mica- $\mathrm{NaCl}$ (aq.) interface. Atomic scale order is seen in $x y, x z$ and $y z$ planes. These 3D maps show the variations of the phase shift of the tip's oscillation. Supplementary Movies 1 (52s), 2 (105s) and 3 (105s) include the complete 3D data, respectively, of a-c. The 3D AFM experiments were performed at $\sim 300 \mathrm{~K}$.

ordered interface that extends several nanometres into the liquid. The ordered structure resembles an epitaxial growth process because the periodicity in the $x y$ plane $(\approx 0.5 \mathrm{~nm})$ follows the periodicity of the mica substrate $(0.52 \mathrm{~nm})$.

The data discussed here is focused on mica-KCl (aq.) interfaces; however, we have included images from a mica- $\mathrm{NaCl}$ (aq.) (Fig. 2c) and mica-RbCl interfaces (Supplementary Fig. 1) to illustrate that the observed phenomenology is not restricted to $\mathrm{KCl}$ solutions. It also applies to other alkali halide electrolyte solutions. The Supplementary Movies 1-3 are associated with the $3 \mathrm{D}$ AFM images from Fig. 2. The movies have been acquired in 52,105 and $105 \mathrm{~s}$, respectively. Those values indicate that the observed ordered liquid layers are stable over several minutes.

Interfacial organization of electrolyte aqueous solutions. The structure, extension and periodicities of the ordered liquid layer depend on the salt concentration. At low to moderate concentrations $(0.01-1 \mathrm{M})$, the 3D AFM data for a $\mathrm{KCl}$ (aq.) solution (Fig. 3a-c) show the presence of adsorbed $\mathrm{K}^{+}$cations on the mica surface and a few hydration layers $(\sim 0.3 \mathrm{~nm}$ thick) above them (Fig. 3a). Figure 3b shows that the force sensed by the tip along the dashed line shown in Fig. 3a alternates repulsive and attractive regions. The curve shows a repulsive region when the tip is on top of an adsorbed cation and two local repulsive maxima $\sim 0.34 \mathrm{~nm}$ apart. To determine the force curve from the $3 \mathrm{D}$ AFM data is complex and tedious because a single force value $F\left(x_{\mathrm{i}}, y_{\mathrm{j}}, z_{\mathrm{k}}\right)$ requires to know the dependence of the observables with $z$ from $z_{\mathrm{k}}$ to $\infty$. We have adapted the force inversion methods deduced for 1D AFM measurements ${ }^{37,38}$. The main steps to deduce the force curve from the 3D AFM observables are illustrated in Supplementary Fig. 2. The measured force depends on the hydration layer order. We report forces between $50-150 \mathrm{pN}$ (Fig. 3b). Those values are comparable to the data provided by molecular dynamics simulations and experiments on a calcite-water interface ${ }^{39}$.

Our CF-DFT calculation reveals the main factors in the above experiments. We do not aim to provide a full quantitative description of the experimental data, nor to give the most realistic description of the ionic solutions, but rather to reproduce and explain the phenomena semi-quantitatively. The theoretical difficulties associated with a realistic description of the ionic interactions are not crucial to predict the qualitative behaviour of the system. The most relevant factor is to observe that by increasing the salt concentration we should get closer to its crystallization phase transition, which may be represented by short-ranged effective interactions. The interaction with the mica is modelled to have a nearly complete monolayer of cations at low salt concentrations $(0.2 \mathrm{M})$ (Fig. $3 \mathrm{e}-\mathrm{g}$ ), as indicated by the AFM images (Fig. 3c). To support the validity of our approach in the Supplementary Information we show that the CF-DFT predictions at high concentration are very robust with respect to a significant reduction of the cation coverage of the mica at low salt concentration (Supplementary Fig. 3). This indicates that the phenomena reported here do not depend on the details of the interactions that explain the high cation coverage as shown in Fig. $3 c^{32-33}$. The oscillations of the water density from the model start with a first layer of water molecules tightly bound to the adsorbed cations and the mica. In the 3D AFM images this first hydration layer cannot be distinguished from the atomic corrugation coming from mica and $\mathrm{K}$ atoms. For that reason it is not marked in Fig. 3a.The following layers, with a separation of about $0.3 \mathrm{~nm}$ in the CF-DFT model, coincide with the local maxima in 3D AFM force and identify with the oscillations observed on the experimental force curve as an effect of the second and third hydration layers. The anions are effectively excluded from the interface.

The experimental $x y$ frames (Fig. 3c,d) reveal the atomic arrangement within the layered structure hinted in the $x z$ frame of the 3D AFM image (Fig. 3a). Figure 3c, at $z=0 \mathrm{~nm}$, shows the hexagonal structure and atomic corrugation of the $\mathrm{K}^{+}$ions (yellow dots, separated $0.52 \mathrm{~nm}$ ) adsorbed on the cleaved mica (001) face. The $\mathrm{K}^{+}$ions from the solution occupy the positions in the cleaved plane previously occupied by $\mathrm{K}^{+}$ions in the bulk crystal; the water molecules from the first hydration layer are tightly bound to the solid surface, occupying the space between the cations. The second hydration layer (Fig. 3d) is placed $0.25 \mathrm{~nm}$ above the adsorbed $\mathrm{K}^{+}$cations and it follows its atomic corrugation $(0.03 \mathrm{~nm})$. Our CF-DFT results agree with this interpretation; the dark hat above each cation represents the local absence of water in the first hydration layer, and the density map (Fig. 3f) gives the clear interpretation that the water molecules in the $2^{\text {nd }}$ layer are centred on top of the cations of the first layer, but with a larger lateral spreading.

At higher salt concentrations $(3-5 \mathrm{M})$, the interfacial layer has a different structure and composition. It is thicker $(\sim 3-4 \mathrm{~nm})$ and shows a crystal-like structure. Figure $4 \mathrm{a}, \mathrm{b}$ show some $x z$ frames taken from 3D AFM images. The observed structure resembles an epitaxial growth because the mean periodicity in the 

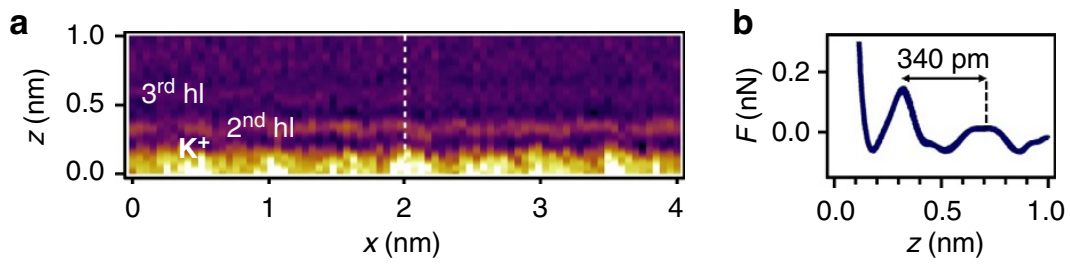

C
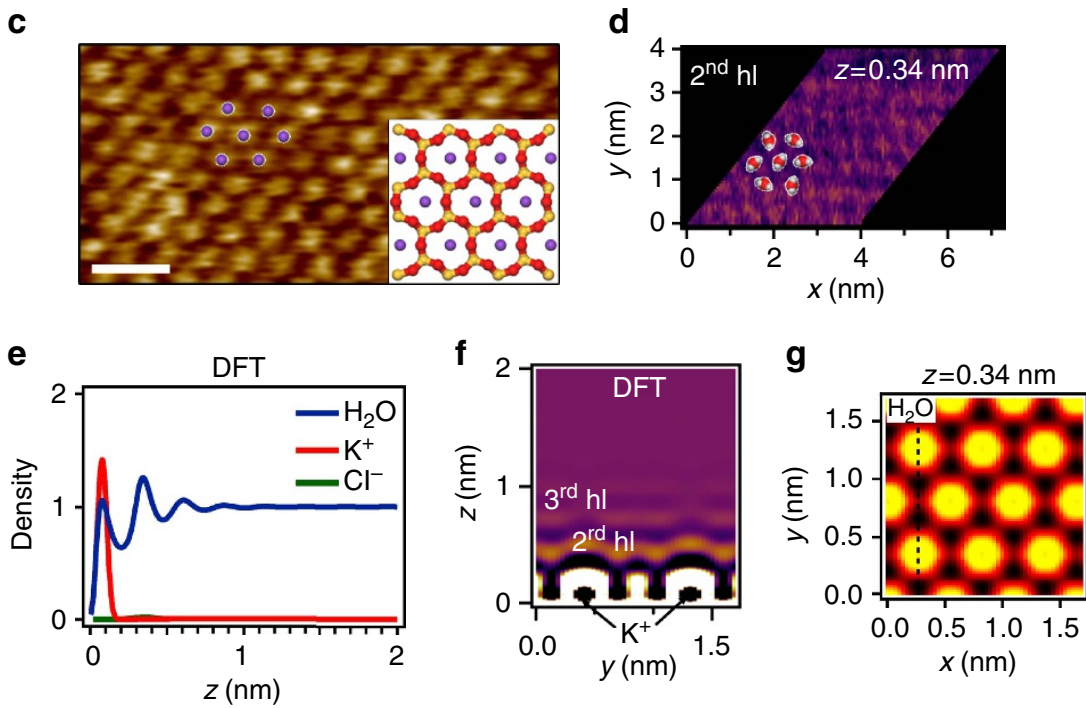

Figure 3 | Atomic scale structure of mica-KCl interfaces at low molarities. (a) $x z$ frame (raw data). At low to moderate salt concentrations (0.01-1 M), the ordered layer is very thin (below $1.0 \mathrm{~nm}$ ). It is formed by a single cation monolayer on top of the mica lattice and a few hydration layers. (b) The force curve across the dashed line of a shows two hydration layers about $0.34 \mathrm{~nm}$ apart. (c) $x y$ frame taken at $z=0 \mathrm{~nm}$. In the experimental images, the $z=0 \mathrm{~nm}$ is chosen to be located at the interstitial position between two $\mathrm{K}^{+}$. This assumption facilitates the direct comparison between theory and CF-DFT data. The image shows the hexagonal structure of the $\mathrm{K}^{+}$on mica. The inset shows the structure of mica (001); Oxygen (red), silicon or aluminium (yellow) and the adsorbed $\mathrm{K}$ ions (purple). Si and $\mathrm{Al}$ are in a 3 to 1 ratio. The plane of the $\mathrm{K}$ atoms lies above the $\mathrm{O}$ and $\mathrm{Si}$ planes. (d) $x y$ frame acquired at $z=0.34 \mathrm{~nm}$. The image shows the structure of the water molecules in the $2^{\text {nd }}$ hydration layer. The origin of $z$ is chosen at the mica surface (minima in $\mathbf{a}$ ). (e) CF-DFT results of the number density perpendicular to the mica surface ( $z$ direction). At each $z$ point, the density profile represents the average value over the corresponding $x y$ plane. The density profiles are normalized with respect to the bulk number density of water. (f) CF-DFT $x z$ total number density map of the mica- $\mathrm{KCl}$ (aq.) interface. This map is calculated for the $x$ position marked with a dashed line in $\mathbf{g}$. (g) CF-DFT $x y$ map at $z=0.34 \mathrm{~nm}$. At this position the map mainly reflects the arrangement of the water molecules. Scale bar, $1 \mathrm{~nm}(\mathbf{c})$.

$x y$ plane is $0.51 \mathrm{~nm}$ (Fig. 4c), which is very close to the mica(001) lattice parameter $(0.52 \mathrm{~nm})$. In the perpendicular direction (Fig. 4d), the force curve shows a decaying oscillatory behaviour. The atomic size features observed in the $x z$ frames indicate the discrete character of the interface in all the spatial coordinates. Remarkably atomic size features are still observed $2 \mathrm{~nm}$ above the mica surface.

The CF-DFT results near a saturated salt concentration (about $5 \mathrm{M}$ in our CF-DFT model) show that the number density profiles for $\mathrm{K}^{+}$and $\mathrm{Cl}^{-}$have strong oscillations as function of the distance from the mica surface (Fig. 4e). Three peaks are clearly observed for each ion, and over that region water molecules are only partially depleted. The contrast mechanism observed in the 3D AFM images at high concentrations is still unknown. The separation among the local maxima in the experimental tip-liquid force (Fig. 4d) is in good agreement with the separation among the local maxima in the net charge profile (Fig. 4f). This observation together with decaying character of the force as a function of the distance from the mica surface suggests a correlation with the global cation-anion 3D structure, rather than with the plain total number density of liquid molecules. The 3D structure in the liquid solution propagates from the mica surface and it cannot be understood without a cooperative effect between the three chemical species. It involves the arrangement of alternating cation and anion planes parallel to the mica surface, still with a significant amount of water, being clearly different from the $\mathrm{KCl}$ crystal that may be obtained in the same CF-DFT model at coexistence with a saturated water solution. As presented in Supplementary Information, the epitaxial crystal found in our CF-DFT model contains very little water inside, as expected in a real $\mathrm{KCl}$ crystal. Thick layers of that epitaxial crystal covering the mica can also be obtained in our CF-DFT calculations, but they imply a strong reorganization of the first density layers, and correspond to separated minima of the grand potential energy.

Experimentally, we also observe the formation of small nanocrystals on the mica at saturation. Supplementary Fig. 4 shows atomic resolution images of $\mathrm{NaCl}$ and $\mathrm{KCl}$ crystals deposited from the solution. They were obtained in the presence of a saturated electrolyte concentration. The complete evaporation of the water from the fluid cell left the mica covered by salt nano or microcrystals (Supplementary Fig. 5). The morphology of the nano and microcrystals follows some of the crystalline orientations of the mica ${ }^{40}$.

\section{Discussion}

Our findings establish three different structures for the interfacial layer formed by alkali halide electrolyte solutions interacting with a mica surface. In pure water and at very low molarities $(\leq 10 \mathrm{mM})$ is very difficult to image the hydration layers. This is due to the diffusive force associated with the negative charge of 


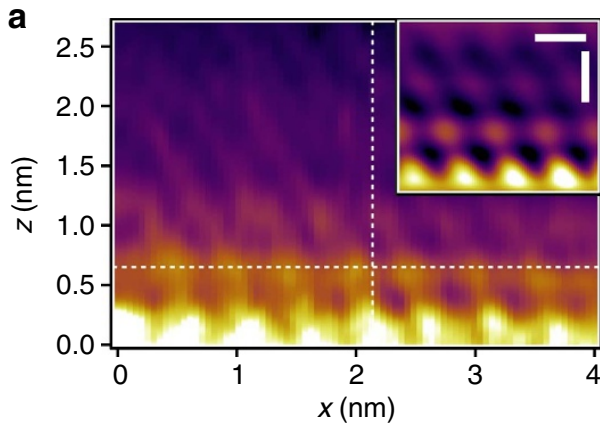

b
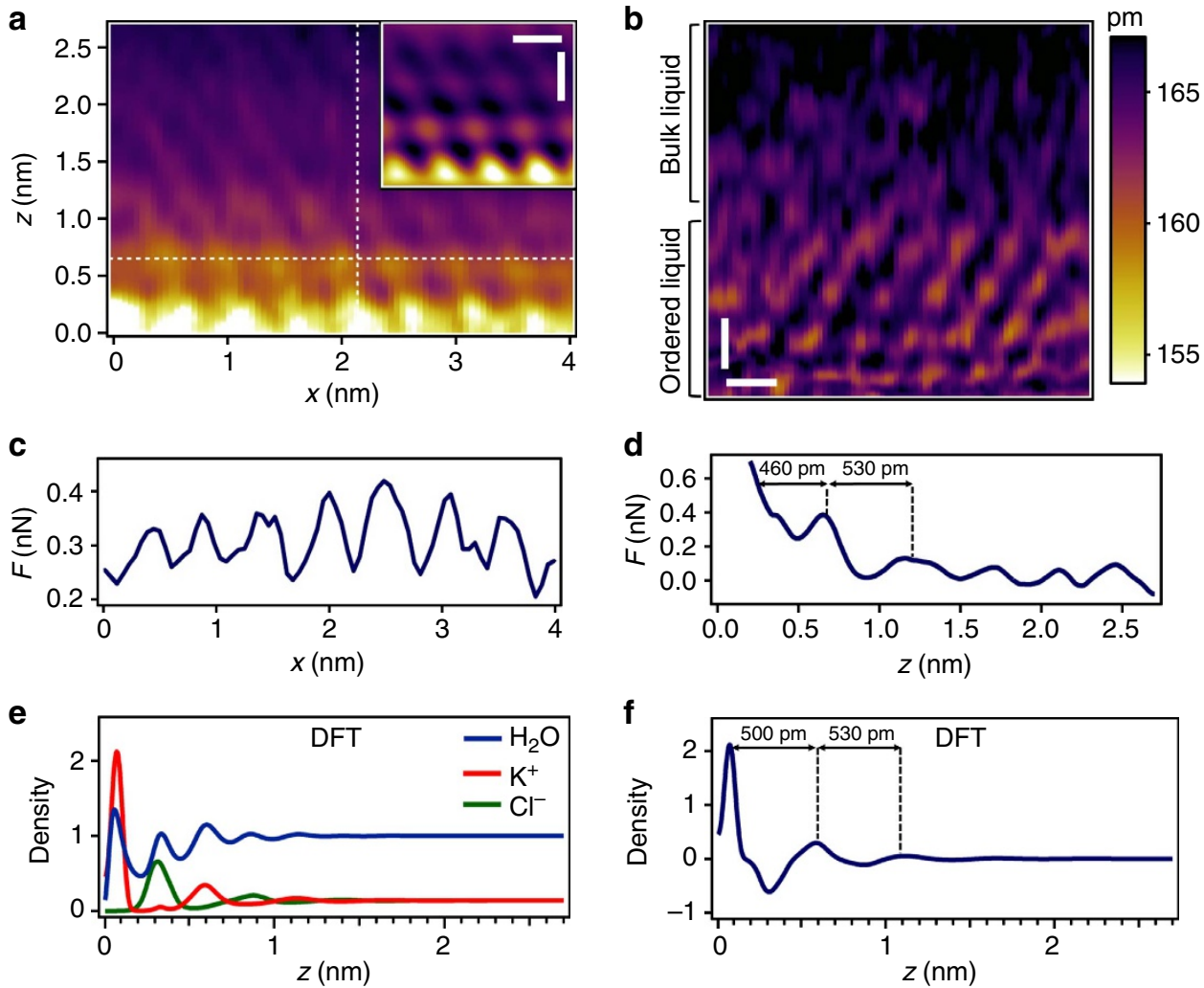

Figure 4 | Atomic scale structure of mica-KCl (aq.) interfaces at high molarities. (a) $x z$ frame (low pass filtered image). At high molarities the interface is characterized by the presence of an ordered liquid layer 2-3 nanometres thick. The inset shows a filtered image (FFT) of the bottom right corner of the $x z$ frame. The ordered layers show atomic scale features along $x$ and $z$ axis. The $x z$ frame shows the variations of the phase shift. (b) $x z$ frame extending $5 \mathrm{~nm}$ above the mica surface (not shown). The side bar shows the variations of the oscillation amplitude during the $z$ motion $\left(A_{0}=169 \mathrm{pm}\right)$. (c) The force curve taken along the horizontal dashed line in a show a mean periodicity of $0.5 \mathrm{~nm}$. It follows the lattice structure of the mica(001). (d) Force curve along the perpendicular line plotted in $\mathbf{a}$. The force oscillates with the distance from the mica. (e) CF-DFT simulations at high molarities. Number density profiles perpendicular to the mica surface for $\mathrm{K}^{+}, \mathrm{Cl}^{-}$and water. The density profiles are normalized with respect to the bulk number density of water. (f) Net charge density profile (cation minus anion). Scale bars, $0.5 \mathrm{~nm}(\mathbf{a}, \mathbf{b})$.

the mica surface. At low molarities, a monolayer of alakali cations is adsorbed on the mica. A few water monolayers are layered above them. At high molarities (3-5 M), an ordered liquid layer several nanometres thick is formed. The lateral periodicity follows the mica lattice parameter while the vertical periodicities are controlled by the interactions between the ions and the water molecules. At saturation, ionic crystals precipitate on the surface of mica.

A central issue of these observations concerns the physical state of the ordered layer, crystalline or liquid. To capture the 3D AFM images, the tip moves up and down into the mica-liquid interface with a frequency of $100 \mathrm{~Hz}$. In the process the tip continuously disrupts and pushes away the ions and water molecules of the ordered layer. This process supports a liquid-like character for the ordered layer. We explain the compatibility of a crystal-like structure with a liquid-like deformability by noting the different time scales involved in the $3 \mathrm{D}$ imaging process and the ion and water molecule motions. A single event in the tip motion happens in the microsecond range, this is, several orders of magnitude larger than the expected times for the motion of individual water molecules and ions $\left(\sim 10^{-10} s\right)$. Thus the 3D AFM images provide the average positions of the ions and water molecules at the millisecond to second time scales.

The role of the tip geometry and composition in atomic resolution experiments in liquid is being studied by molecular dynamics simulations ${ }^{41}$. Ions and hydration layers should also be formed on the tip. However, the ordering of the tip's interfacial layer should be weaker than the one found on mica because the tip's nanoscale roughness. We propose that the 3D AFM images represent the structure of the ordering of electrolytes on the mica with little distortion from the tip. This interpretation is consistent with the agreement observed between the experiments and the theory, which represents the equilibrium 3D density distributions in the unperturbed mica-liquid interface.

Force microscopy has provided atomic and molecular resolution images of a large variety of surfaces from crystalline systems $^{42}$ to biomolecules ${ }^{43}$. The combination of atomic resolution with a genuine $x y z$ probe motion opens the way to investigate the $3 \mathrm{D}$ atomic structure and interactions of ions and solvent molecules near solid surfaces with a few nanometres depth. These findings could serve as the model for the structure of electrolyte interfacial layers in the vicinity of hydrophilic surfaces with relevance in a large variety of fields from surface electrochemistry to molecular and cell biology.

\section{Methods}

3D AFM. The 3D AFM images of solid-liquid interfaces were obtained by introducing a sequential 3D motion for the tip. This motion is independent of the tip oscillation. The 3D tip motion involves a sinusoidal signal added to piezo $z$, and a modification of the $x y$ tip motion to acquire data points on either of trace or retrace but not in both. The 3D AFM was operated in amplitude modulation $\mathrm{AFM}^{35}$ by using a 'loose' feedback control. This has been achieved by decreasing the bandwidth of the $z$ feedback loop between $1-2 \mathrm{kHz}$. This enables tip-sample plane tilt correction without affecting the sinusoidal movement. The free oscillation amplitudes $A_{0}$ were in the $35-190 \mathrm{pm}$ range and the set-point amplitude was set at 
a $0.9-0.95$ of $A_{0}$. The oscillation of the cantilever was driven by photothermal excitation at a wavelength of $405 \mathrm{~nm}$. The 3D AFM was implemented in a commercial AFM platform (Cypher, Asylum Research, Oxford Instruments).

3D motion. The AFM images involve a maximum frequency of $50 \mathrm{kHz}$ per data point. For images with a $z$ depth equal or below $1 \mathrm{~nm}$ (Fig. 2a), the $z$ displacement is performed at a frequency of $100 \mathrm{~Hz}$ with a sampling performed in both directions, approach and retract from the sample (512 data points in total); the $x$ displacement is performed with a frequency of $1.22 \mathrm{~Hz}$ and the sampling involves 80 data points; the $y$ displacement is performed with a frequency of $0.02 \mathrm{~Hz}$ and the sampling involves 64 data points. For 3D images with a $z$ depth above $2 \mathrm{~nm}$ (Fig. 2b,c), the $z$ displacement is performed at a frequency of $50 \mathrm{~Hz}$ with a sampling of 1,024 data points (512 approach and 512 retract); the $x$ displacement is performed at a frequency of $0.61 \mathrm{~Hz}$ with a sampling of 80 data points and the $y$ displacement is performed at a frequency of $0.01 \mathrm{~Hz}$ with a sampling of 64 data points.

Microcantilevers. We have used silicon cantilevers PPP_NCHAu_D (Nanosensor, Germany). The force constant, resonant frequency and quality factors of the first two eigenmodes were determined in liquid. Their values were in the following range, $k_{1}=22-40 \mathrm{~N} \mathrm{~m}^{-1} f_{1}=120-180 \mathrm{KHz}, k_{2}=800-1,600 \mathrm{~N} \mathrm{~m}^{-1}$ $f_{2}=800-1,100 \mathrm{KHz}, Q_{1}=7-12, Q_{2}=18-25$. The above values have been determined in water. The force constants have been determined by using the thermal method.

Sample preparation. Discs of muscovite mica (SPI supplies, USA) were cleaved with adhesive tape before each experiment. For aqueous $\mathrm{NaCl}$ solution experiments, the muscovite mica was thoroughly rinsed with pure water $(18.2 \mathrm{M} \Omega)$ before introducing it in the AFM chamber. $\mathrm{NaCl}$ and $\mathrm{KCl}$ solutions were prepared from $99.5 \%(\mathrm{NaCl})$ and $99.0 \%(\mathrm{KCl})$ salts (Sigma-Aldrich) dissolved in ultrapure water $(18.2 \mathrm{M} \Omega, \mathrm{pH}=5.5)$.

Classical Fluid Density Functional Theory. We use the DF formalism, which is the most successful theoretical framework to get a consistent description of the molecular structure and the thermodynamics of interfacial liquid systems ${ }^{14,44}$. We model the system as a ternary liquid mixture, with a solvent (water) as major component (bulk density $\rho_{s}$ ), and equal concentrations of two solute species (cations and anions), with equal bulk densities, $\rho_{c}=\rho_{a}$, given by the fraction $X=\rho_{c, a} / \rho_{t}$ over the total density. The molecular packing effects are obviously at the core of the 3D molecular structure, and they are included in our model as Hard Sphere impenetrability of the molecules. We have taken different Hard Sphere diameters for the three species; for the ions twice its ionic radius ${ }^{45}, \sigma_{\mathrm{Cl}}=0.362$ and $\sigma_{\mathrm{K}}=0.266$ and $\sigma \equiv \sigma_{\text {Water }}=0.2569 \mathrm{~nm}$ for the water. In a previous simplified model we had used the same molecular diameter for all the species, $\sigma$, that fixes the length scale in the model, and the good qualitative comparison between the two models provides an important clue. The size differences between the ions, as well as the anisotropy of water, would certainly be important for a quantitative prediction, but as shown by our results, these effects are not needed to understand the qualitative aspects of the experimental observations. What is crucial is to have a good DF description of the molecular packing for the extremely inhomogeneous density distributions of the first cation layer on the mica. Therefore, we use the dimensional interpolation fundamental measure theory-free energy, which reproduces very accurately the crossover from $0 \mathrm{D}$ cavities, to $3 \mathrm{D}$ bulk liquids and to the crystallization of the Hard Sphere system ${ }^{46,47}$. Additional description is provided in Supplementary Figs 6-7 and Supplementary Methods.

Data availability. The data that support the findings of this study are available from the corresponding author on request.

\section{References}

1. Verdaguer, A., Sacha, G. M., Bluhm, H. \& Salmeron, M. Molecular structure of water at interfaces: wetting at the nanometer scale. Chem. Rev. 106, 1478-1510 (2006).

2. Li, Q., Song, J., Besenbacher, F. \& Dong, M. D. Two-dimensional material confined water. Accounts Chem. Res. 48, 119-127 (2015).

3. Voitchovsky, K., Kuna, J. J., Contera, S. A., Tosatti, E. \& Stellacci, F. Direct mapping of the solid-liquid adhesion energy with subnanometre resolution. Nat. Nanotechnol. 5, 401-405 (2010).

4. Collins, L. et al. Probing charge screening dynamics and electrochemical processes at the solid-liquid interface with electrochemical force microscopy. Nat. Commun. 5, 3871 (2014).

5. Li, T. D., Gao, J., Szoszkiewicz, R., Landman, U. \& Riedo, E. Structured and viscous water in subnanometer gaps. Phys. Rev. B 75, 115415 (2007)

6. Garcia, R., Knoll, A. W. \& Riedo, E. Advanced scanning probe lithography. Nat. Nanotechnol. 9, 577-587 (2014).
7. Spitzner, E. C., Röper, S., Zerson, M., Bernstein, A. \& Magerle, R. Nanoscale swelling heterogeneities in type I collagen fibrils. ACS Nano 9, 5683-5694 (2015).

8. Bizzarri, A. R. \& Cannistraro, S. Molecular dynamics of water at the protein-solvent interface. J. Phys. Chem. B 106, 6617-6633 (2002).

9. Levy, Y. \& Onuchic, J. N. Water mediation in protein folding and molecular recognition. Ann. Rev. Biophy. Biomol. Struct. 35, 389-415 (2006).

10. Israelachvili, J. \& Wennerstrom, H. Role of hydration and water structure in biological and colloidal interactions. Nature 379, 219-225 (1996).

11. Butt, H. J. \& Kappl, M. Surface and Interfacial Forces 117-119 (Wiley-VCH Verlag GmbH \& Co. KGaA, 2010).

12. Davidchack, R. L. \& Laird, B. B. Crystal structure and interaction dependence of the crystal-melt interfacial free energy. Phys. Rev. Lett. 94, 4 (2005).

13. Bauer, C., Dietrich, S. \& Parry, A. O. Morphological phase transitions of thin fluid films on chemically structured substrates. Europhys. Lett. 47(4): 474-480 (1999).

14. Lowen, H. Density functional theory of inhomogeneous classical fluids: recent developments and new perspectives. J. Phys. Cond. Matter 46, 11897-11905 (2002).

15. Israelachvili, J. N. \& Pashley, R. M. Molecular layering of water at surfaces and origin of repulsive hydration forces. Nature 306, 249-250 (1983).

16. Tarazona, P. \& Vicente, L. A model for density oscillations in liquids between solid walls. Mol. Phys. 56, 557-572 (1985).

17. O'Shea, S. J. \& Welland, M. E. Atomic force microscopy at solid-liquid interfaces. Langmuir 14, 4186-4197 (1998).

18. Fenter, P. \& Lee, S. S. Hydration layer structure at solid-water interfaces. MRS Bull. 39, 1056-1061 (2014).

19. Velasco-Velez, J. J. et al. The structure of interfacial water on gold electrodes studied by x-ray absorption spectroscopy. Science 346, 831-834 (2014).

20. Ulcinas, A. et al. Shear Response of Nanoconfined Water on Muscovite Mica: Role of Cations. Langmuir 27, 10351-10355 (2011).

21. Labuda, A., Kobayashi, K., Suzuki, K., Yamada, H. \& Grütter, P. Monotonic damping in nanoscopic hydration experiments. Phys. Rev. Lett. 110, 066102 (2013).

22. Feibelman, P. J. The first wetting layer on a solid. Phys. Today 63, 34-39 (2010).

23. Reischl, B., Watkins, M. \& Foster, A. S. Free energy approaches for modeling atomic force microscopy in liquids. J. Chem. Theory Comp. 9, 600-608 (2013).

24. Leng, Y. Hydration force between mica surfaces in aqueous $\mathrm{KCl}$ electrolyte solution. Langmuir 28, 5339-5349 (2012).

25. Toth, G. I., Tegze, G., Pusztai, T. \& Granasy, L. Heterogeneous crystal nucleation: the effect of Lattice Mismatch. Phys. Rev. Lett. 108, 025502 (2012).

26. Fukuma, T., Ueda, Y., Yoshioka, S. \& Asakawa, H. Atomic-scale distribution of water molecules at the mica-water interface visualized by three-dimensional scanning force microscopy. Phys. Rev. Lett. 104, 016101 (2010).

27. Kimura, K. et al. Visualizing water molecule distribution by atomic force microscopy. J. Chem. Phys. 132, 194705 (2010).

28. Arai, T. et al. Atom-resolved analysis of an ionic $\mathrm{KBr}(001)$ crystal surface covered with a thin water layer by frequency modulation atomic force microscopy. Langmuir 31, 3876-3883 (2015).

29. Marutschke, C. et al. Three-dimensional hydration layer mapping on the (10.4) surface of calcite using amplitude modulation atomic force microscopy. Nanotechnology 25, 335703 (2014).

30. Atsakawa, H., Yoshioka, S. \& Fukuma, T. Spatial distribution of lipid headgroups and water molecules at membrane/water interfaces visualized by three-dimensional scanning force microscopy. ACS Nano 6, 9013-9020 (2012).

31. Herruzo, E. T., Asakawa, H., Fukuma, T. \& Garcia, R. Three-dimensional quantitative force maps in liquid with 10 piconewton, angstrom and sub-minute resolutions. Nanoscale 5, 2678-2685 (2013).

32. Siretanu, I. et al. Direct observation of ionic structure at solid-liquid interfaces: a deep look into the Stern Layer. Sci. Rep. 4, 4956 (2014).

33. Ricci, M., Spijker, P. \& Voitchovsky, K. Water-induced correlation between single ions imaged at the solid-liquid interface. Nat. Commun. 5, 4400 (2014).

34. Loh, S. H. \& Jarvis, S. P. Visualization of ion distribution at the mica-electrolyte interface. Langmuir 26, 9176-9178 (2010).

35. Araki, Y. et al. Direct observation of the influence of additives on calcite hydration by frequency modulation atomic force microscopy. Cryst. Growth Des. 14, 6254-6260 (2014).

36. Garcia, R. \& San Paulo, A. Attractive and repulsive tip-sample interaction regimes in tapping mode AFM. Phys. Rev. B 60, 4961-4967 (1999).

37. Holscher, H. Quantitative measurement of tip-sample interactions in amplitude modulation atomic force microscopy. Appl. Phys. Lett. 89, 3 (2006).

38. Payam, A. F., Martin-Jimenez, D. \& Garcia, R. Force reconstruction from tapping mode force microscopy experiments. Nanotechnology 26, 185706 (2015).

39. Fukuma, T. et al. Mechanism of atomic force microscopy imaging of three-dimensional hydration structures at a solid-liquid interface. Phys. Rev. B 92, 7 (2015). 
40. Wu, J. H., Ang, S. G. \& Xu, G. Q. Atomic force microscopy study of self-assembled sodium chloride nanocrystallites and their morphology transitions. J. Phys. Chem. C 112, 7605-7610 (2008).

41. Rischl, B., Watkins, M. \& Foster, A. S. Free energy approaches for modeling atomic force microscopy in liquids. J. Chem. Theory Comput. 9, 600-608 (2013).

42. Barth, C., Foster, A. S., Henry, C. R. \& Shluger, A. L. Recent Trends in surface characterization and chemistry with high-resolution scanning force methods. Adv. Mater. 23, 477-501 (2011).

43. Bippes, C. A. \& Muller, D. J. High-resolution atomic force microscopy and spectroscopy of native membranes. Rev. Prog. Phys. 74, 086601 (2011).

44. Evans, R. The nature of the liquid-vapour interface and other topics in the statistical mechanics of non-uniform, classical fluids. Adv. Phys. 28, 143 (1979).

45. Ashcroft, N. W. \& Nermin, N. D. Solid State Physics (Holt, Rinehart and Winston, 1976).

46. Tarazona, P. Density functional for hard spheres crystals: a fundamental measure approach. Phys. Rev. Lett. 84, 694 (2000).

47. Tarazona, P., Cuesta, J. A. \& Martinez-Ratón, Y. in Lecture Notes in Physics. (ed. Mulero, A.) 753, 247-341 (Springer, 2008).

\section{Acknowledgements}

We thank helpful discussions with Dr Mingdong Dong. This work was funded by the European Research Council ERC-AdG-340177 (3DNanoMech) and Spanish Ministry of Science and Innovation (CSD2010-00024, FIS2013-47350-C5, MDM-2014-0377).

\section{Author contributions}

D.M.-J. and R.G. planned the experiments. D.M.-J. performed the experiments. E.C. and P.T. performed the FDT calculations. R.G. conceived the 3D AFM method and wrote the manuscript. All authors analysed and discussed the data.

\section{Additional information}

Supplementary Information accompanies this paper at http://www.nature.com/ naturecommunications

Competing financial interests: The authors declare no competing financial interests.

Reprints and permission information is available online at http://npg.nature.com/ reprintsandpermissions/

How to cite this article: Martin-Jimenez, D. et al. Atomically resolved three-dimensional structures of electrolyte aqueous solutions near a solid surface. Nat. Commun. 7:12164 doi: 10.1038/ncomms12164 (2016).

(c) (i) International License. The images or other third party material in this article are included in the article's Creative Commons license, unless indicated otherwise in the credit line; if the material is not included under the Creative Commons license, users will need to obtain permission from the license holder to reproduce the material. To view a copy of this license, visit http://creativecommons.org/licenses/by/4.0/

(C) The Author(s) 2016 